University of South Carolina

Scholar Commons

3-25-2005

\title{
Semiclassical Nonadiabatic Dynamics with Quantum Trajectories
}

Vitaly A. Rassolov

University of South Carolina - Columbia, rassolov@mailbox.sc.edu

Sophya V. Garashchuk

University of South Carolina - Columbia, garashch@mailbox.sc.edu

Follow this and additional works at: https://scholarcommons.sc.edu/chem_facpub

Part of the Chemistry Commons

\section{Publication Info}

Physical Review A, Volume 71, 2005, pages 032511-.

(c) Physical Review A 2005, American Physical Society.

This Article is brought to you by the Chemistry and Biochemistry, Department of at Scholar Commons. It has been accepted for inclusion in Faculty Publications by an authorized administrator of Scholar Commons. For more information, please contact digres@mailbox.sc.edu. 


\title{
Semiclassical nonadiabatic dynamics with quantum trajectories
}

\author{
Vitaly A. Rassolov and Sophya Garashchuk \\ Department of Chemistry and Biochemistry, University of South Carolina, Columbia South Carolina 29208, USA
}

(Received 19 November 2004; revised manuscript received 20 January 2005; published 25 March 2005)

\begin{abstract}
Dynamics based on quantum trajectories with approximate quantum potential is generalized to nonadiabatic systems and its semiclassical properties are discussed. The formulation uses the mixed polar-coordinate space representation of a wave function. The polar part describes the overall time evolution of the wave-function components semiclassically using the single-surface approximate quantum potential. The coordinate part represents a complex"population" amplitude, which in case of localized coupling can be solved for quantum mechanically in an efficient manner. In the high-energy regime this is accomplished by using a small basis determined by the coupling between surfaces. An illustration is given for a typical curve-crossing problem. The energy-resolved probabilities obtained from the time evolution of two wave packets for a wide range of energies are in excellent agreement with exact results for energies above the threshold of the diabatic reaction, including the case of total nonadiabatic transition.
\end{abstract}

DOI: $10.1103 /$ PhysRevA.71.032511

PACS number(s): 31.50.Gh

\section{INTRODUCTION}

Quantum-mechanical (QM) effects in molecular dynamics are essential for accurate description and understanding of many chemical processes, such as those in gas-phase and surface reactions, in photochemistry, in interactions of molecules with electric fields, and in the chemistry of polymers, clusters, and liquids. QM effects are most pronounced in processes involving hydrogen atoms, including reactions in enzymes and in other biomolecular environments. Proton transfer through a hydrogen bond is a widespread phenomenon relevant to fields ranging from materials science to biology, present, for example, in DNA-based molecular wires [1] and water wires in membrane protein channels [2]. Enzyme catalysis in living systems is affected by modulation of the strength of the hydrogen bonds and even by the promotion of hydrogen tunneling [3]. Quantum effects are obviously important in other areas as well, such as astronomy, combustion, and atmospheric chemistry. For example, the anomalous isotope effect in ozone formation-a preference for heavier species compared to ${ }^{16} \mathrm{O}_{3}$ - can be attributed to the long-lived resonance states in ozone [4].

Arguably, nonadiabatic dynamics is the most important quantum effect on the motion of nuclei in chemical reaction dynamics including reactive scattering, photochemistry, and enzymatic reactions [5-7]. Electron and proton transfer in chemical and biological systems [8] and nonradiative processes in solids and on solid surfaces, such as molecular desorption and ion neutralization [9], involve several potential surfaces with transitions among them. While some nonadiabatic processes can be described by the time-independent or time-dependent Schrödinger equation (SE), molecular processes in laser fields with laser-induced curve crossings [10] are examples of intrinsically time-dependent nonadiabatic transition problems.

Exact methods of solving the time-dependent SE based on the grid or basis function representation are unfeasible for systems beyond 10-12 degrees of freedom, because of the exponential scaling of numerical efforts with the system size.
The current state-of-the-art full-dimensional quantum studies are limited to a few tetra-atomic reactions and to the $\mathrm{CH}_{4}$ $+\mathrm{H} \rightarrow \mathrm{CH}_{3}+\mathrm{H}_{2}$ reaction [11-13]. At the present, essentially all molecular dynamics simulations, which are routinely used to study large molecular systems, use only classical mechanics, which has two fundamental limitations: (i) the classical motion of nuclei; (ii) the Born-Oppenheimer separation of motion of electrons and nuclei resulting in a single-electronic surface dynamics. Therefore, cheap yet accurate and systematically improvable means of including dominant quantum effects into molecular dynamics are highly desirable. Recently, we have suggested an approximate propagation method based on quantum trajectories, described in Sec. II, which proved to be efficient and accurate for model chemical systems. Generalization of this approach to nonadiabatic dynamics consistent with the semiclassical (SC) propagation is presented in Sec. III. Numerical application to a curvecrossing model of Tully [14] and discussion are presented in Sec. IV. Section V concludes.

\section{SEMICLASSICAL DYNAMICS BASED ON QUANTUM TRAJECTORIES}

\section{A. The quantum trajectory formulation}

Over the years great efforts went into development of semiclassical time-dependent methods. These methods combine favorable scaling of classical trajectory methods with the description of the dominant quantum effects in the limit of large mass, appropriate for description of nuclei. The traditional SC methods, out of which the initial-value representation methods [15-17] are currently the most popular, are based on the $\hbar \rightarrow 0$ limit of the SE. They involve a purely classical time evolution of trajectories sampling the phase space, while the quantum effects come from summation over the classical paths using the action function and stability of classical trajectories. Despite many improvements in methodology and several impressive high-dimensional applications $[18,19]$, the implementation of such methods remains expensive, the convergence with respect to the number of 
trajectories is poor, and the quality of the description is hard to estimate or improve. Recently we suggested a different SC approach based on the hydrodynamic or de Broglie-Bohm formulation of the SE [20]. This formulation is formally equivalent to the exact quantum mechanics, and the solution can be represented in terms of quantum trajectories. The basic equations presented here, for simplicity, for a particle of mass $m$ moving in a potential $V$ in one dimension, are based on the polar form of the wave function,

$$
\psi(x, t)=A(x, t) \exp \left(\frac{i}{\hbar} S(x, t)\right)
$$

substituted into the SE. [Single-valuedness of $\psi(x, t)$ implies that quantum trajectories should not cross.] The amplitude $A(x, t)$ or the density $\rho(x, t)=A^{2}(x, t)$ and the phase $S(x, t)$ of the wave function are determined along the quantum trajectories specified by position $x$ and momentum $p$, evolving in time according to Hamilton's equations of motion,

$$
\frac{d x}{d t}=\frac{p}{m}, \quad \frac{d p}{d t}=-\frac{\partial(V+U)}{\partial x},
$$

with the definitions

$$
p=\frac{\partial S(x, t)}{\partial x}, \quad U=-\frac{\hbar^{2}}{2 m} \frac{A^{\prime \prime}(x, t)}{A(x, t)} .
$$

A prime denotes a derivative with respect to $x$. In contrast to classical trajectories, quantum trajectories evolve in the presence of an additional quantum potential $U$. The phase $S(x, t)$ is simply the action function along a trajectory. The density $\rho(x, t)$ satisfies the usual continuity equation

$$
\frac{d S(x, t)}{d t}=\frac{p^{2}}{2 m}-(V+U), \quad \frac{d \rho(x, t)}{d t}=-\frac{p^{\prime}}{m} \rho(x, t) .
$$

The full time derivative is defined along a trajectory as

$$
\frac{d}{d t}=\frac{\partial}{\partial t}+\frac{p}{m} \frac{\partial}{\partial x} .
$$

The initial positions for the quantum trajectories sample the coordinate space according to $\rho(x, 0)$. This trajectory description is very compact-it can be interpreted as the most efficient time-dependent "self-adjusting" grid. The quantum behavior is effectively compressed into a single quantity, the nonlocal quantum potential. Quantum trajectories provide intuitive interpretation and visualization tools [21], and the formalism has been extended to the density-matrix approaches and phase-space formulations [22-28].

In recent years the quantum trajectory propagation method has gained attention as an alternative to the traditional QM methods, and several approaches based on the local interpolation of the wave-function density proved to be efficient for model problems in many dimensions [29,30]. For general problems the accuracy of the quantum potential and consequently that of the dynamics was found to deteriorate with time, because of the singularities in the quantum force near the density nodes, often manifested in crossing of the quantum trajectories. This led to the development of several interesting approaches, such as representation transfor- mation, adaptive moving grids, artificial viscosity techniques, covering functions, and counterpropagating waves [31-35]. Independent-trajectory approximations based on the derivative propagation $[36,37]$ and on the stability matrix of trajectories [38], insensitive to the node problem, have also been suggested.

\section{B. Approximate quantum potential}

We consider the quantum trajectory formulation as the starting point for a well-defined semiclassical propagation method using an approximate quantum potential (AQP) $[39,40]$. The SC framework enables us to describe leading quantum effects on dynamics by averaging over the wavefunction density, including possible singularities in the exact quantum potential, and ensures stable dynamics. In Sec. II C we define the term "semiclassical dynamics" in the context of quantum trajectories, and discuss its general features. The classical limit can be defined as the nonclassical momentum (and, consequently, quantum potential) being negligible on the entire space. When the AQP is determined with high accuracy, the formulation becomes equivalent to the full quantum mechanics. In the context of nuclear dynamics, we are interested in the intermediate regime when the AQP is simple enough to be determined globally, making it efficient and practical in many dimensions, yet it is accurate enough to describe leading quantum effects in SC systems. We specified a class of energy-conserving AQPs, with the simplest physically meaningful AQP being quadratic on the entire space [41] or on subspaces [42]. Parameters of the quadratic $\mathrm{AQP}$ on the full space are obtained from linearization of the nonclassical component $r(x, t)$ Eq. (11) of the momentum operator by minimizing the functional

$$
I=\int[r(x, t)-g(x)]^{2} \rho(x, t) d x_{t}
$$

with respect to parameters $\left\{a_{0}, a_{1}\right\}$ of a linear function $g(x)$ $=a_{0}+a_{1} x$. This function defines the following AQP:

$$
U=-\frac{\hbar^{2}}{2 m}\left[g^{2}(x)+g^{\prime}(x)\right] .
$$

The minimization problem, including its multidimensional generalization, is solved by means of linear algebra. Remarkably, the only addition to the classical trajectory propagation is computation of the first and second moments of the trajectory distribution.

Besides AQP, another distinct feature of our approach, is the use of trajectory weights instead of solving the continuity equation (4). The weights are defined as the amount of density within the volume element $\Omega(x, t)$ associated with each trajectory. For closed systems the weights remain constant in time [43],

$$
w=\rho(x, t) \Omega(x, t), \quad \frac{d w}{d t}=0,
$$

which leads to a numerically stable, efficient, and more accurate propagation. Formally, conservation of trajectory weights means that trajectories with zero initial weight can- 
not contribute at later times and, therefore, quantum trajectories with nonzero weights represent the wave function at all times in a compact manner. Using weights also allows one to perform Monte Carlo sampling for high-dimensional systems and to evaluate integrals in Eq. (6) and expectation values, in general, as sums over trajectories. Computation of other quantities, such as wave-function overlaps, is discussed in Ref. [39].

The simplest form of $\mathrm{AQP}$ is already quite promising: (i) it is exact for Gaussian wave packets in locally quadratic potentials - a widely used representation of moving nuclei; (ii) it describes dominant QM effects, such as tunneling, zero-point energy, and energy redistribution; (iii) it is stable and cheap with essentially linear scaling of the numerical effort with the system size; (iv) it can be taken to the exact QM limit using subspaces [42] or using $g(x)$ expandable in a complete (in practice, large) basis. Our calculations for the two-dimensional models of ICN photodissociation [41] and for the hydrogen exchange reactions [42] give accurate SC wave-packet probabilities and spectra, and are faster than the standard QM propagation on a grid and the SC initial-value representation calculation [44].

\section{Semiclassical conditions}

The quantum trajectory formulation of the SE gives a different perspective on the term "semiclassical dynamics." The traditional SC condition is based on the WKB approximation to solutions of the time-independent SE. This condition states that the action function must be much larger than Planck's constant, where the classical momentum $p$ is defined as

$$
p=\sqrt{2 m(E-V)}, \quad \int p d x \gg \hbar .
$$

Application of the QM momentum operator to a wave function in polar form (1),

$$
-i \hbar \frac{\partial}{\partial x} \psi(x, t)=\left(-i \hbar \frac{A^{\prime}(x, t)}{A(x, t)}+S^{\prime}(x, t)\right) \psi,
$$

suggests an alternative criterion. Since the second term in parentheses is the Bohmian trajectory momentum analogous to a classical one [Eq. (3)], the quantity

$$
r=\frac{A^{\prime}(x, t)}{A(x, t)}
$$

can be interpreted as the nonclassical momentum. Then, the $\mathrm{SC}$ condition can be stated as

$$
\hbar|r| \ll|p|,
$$

or in terms of energy, given that $U=-\hbar^{2}\left(r^{2}+r^{\prime}\right) /(2 m)$, as

$$
|U| \ll \frac{p^{2}}{2 m} .
$$

We find that the momentum condition (12) is more convenient than Eq. (13), because the former is expressed in terms of simple quantities which are linear in the semiclas- sical picture of a moving particle, i.e., for Gaussian wave packets.

In the context of the trajectory dynamics the momentum $\mathrm{SC}$ condition (12) is more general than the WKB expression (9), since it is not based on a particular approximate solution to the SE. Moreover, the condition (12) is more convenient, because it is expressed in terms of $r$ and $p$ which are natural quantities in the Bohmian formulation. At the same time, these conditions are closely related, since for semiclassical systems, for which $U$ is small, the Bohmian momentum given by Eqs. (2) and (3) and the WKB momentum are close to each other.

According to Eq. (12) the semiclassical approximation breaks down near the wave-function nodes where $A(x, t)=0$ (the so-called "node problem"). This leads to singular forces acting on quantum trajectories, causing numerical instabilities. A similar breakdown in the WKB approximation occurs near classical turning points. In the context of purely classical trajectory dynamics, this problem was dealt with by developing uniform semiclassical methods $[16,45]$. A generalpurpose semiclassical method based on quantum trajectories must also satisfy Eq. (12) in a uniform sense, i.e., for all points in coordinate space. Therefore, for semiclassical propagation we use the approximate quantum potential outlined in Sec. II B, defined through the linearization of the nonclassical momentum via averaging over the wavefunction density. The relevant SC condition becomes

$$
\hbar\langle|r|\rangle \ll\langle|p|\rangle .
$$

The singularities in $r$ have negligible contributions to the dynamics due to vanishing wave-function density. The average values are defined as $\langle F\rangle=\int F A^{2}(x, t) d x$.

A general semiclassical method must satisfy the SC condition at all times during time evolution, and therefore cannot be defined for a specific form of initial wave functions. In practice, this means that any approximations of the method must be made only for quantities that are negligible in the $m \rightarrow \infty$ or $\hbar \rightarrow 0$ limit. In particular, if the singularities in quantum potential are removed by constraining the density, phase, or momentum, this would generally violate the SC condition. The AQP method constrains the functional form of the nonclassical momentum $r(x, t)$, which enters the SC condition (12) with the $\hbar$ prefactor, while keeping the density itself unconstrained.

Traditional semiclassical methods, such as WKB and Van Vleck-Gutzwiller propagator [46-48], are defined through the $\hbar$ expansion of the solution to the SE converging to the exact result. Methods that are not based on analytic solutions, such as the AQP method, can be considered semiclassical if they are systematically improvable in the limit of large mass for arbitrary physically reasonable initial wave function and kinetic energy density. The kinetic energy density is defined as $-\hbar^{2} \nabla^{2} \psi /(2 m \psi)$. "Systematically improvable," as commonly used, means that there is a general unambiguous numerical prescription for convergence toward the exact solution. The AQP approach is systematically improvable if the linearization of the nonclassical momentum is 


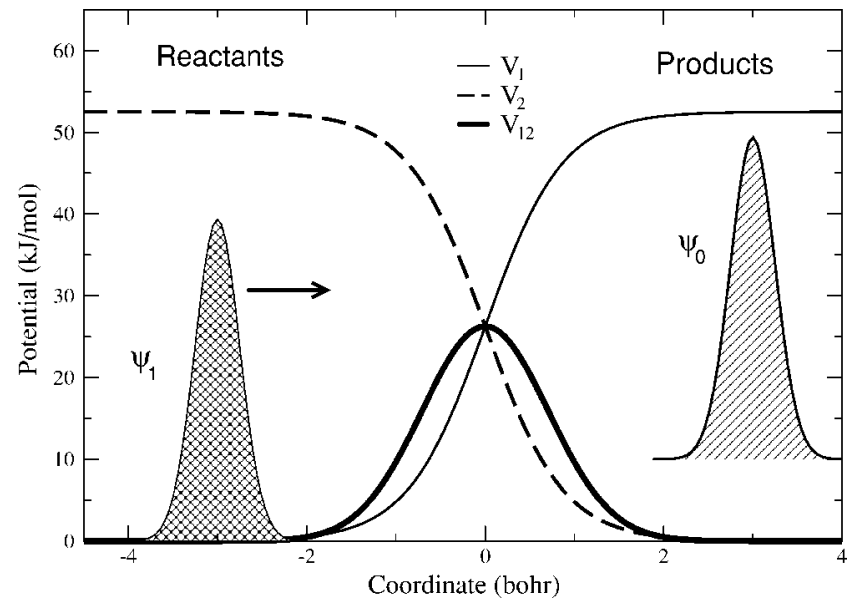

FIG. 1. The curve-crossing model: diabatic potentials $V_{1}$ and $V_{2}$ and coupling $V_{12}$ are shown with the thin solid, dashed, and thick solid lines, respectively. The wave packet $\psi_{1}$ initially located in the reactant region of $V_{1}$ is propagated toward the product region. Analysis of its overlap with a stationary wave packet $\psi_{0}$ gives the energy-resolved reaction probabilities.

accomplished over subspaces [42] or if $r(x, t)$ is represented in terms of a complete basis.

Though the AQP formulation is exact in principle, it becomes impractical beyond the SC regime, namely, when behavior of a system is dominated by interference and nonadiabatic effects. We found that a mixed wave-function representation can efficiently describe interference within the nonsingular dynamics of the modified quantum trajectories [49]. Generalization of the AQP method including the mixed representation approach is the basis for the semiclassical description of nonadiabatic dynamics described below.

\section{SEMICLASSICAL NONADIABATIC DYNAMICS}

\section{A. The Bohmian formulation}

We consider a wave packet evolving in the presence of two one-dimensional potential surfaces $V_{1}$ and $V_{2}$ coupled by a potential $V_{12}$. A typical curve-crossing system in the diabatic representation is sketched on Fig. 1. A multidimensional generalization in Cartesian coordinates is straightforward. This system is described by a two-component wave function $\psi=\left\{\psi_{1}, \psi_{2}\right\}$, governed by the time-dependent SE,

$$
\begin{aligned}
& \left(-\frac{\hbar^{2}}{2 m} \frac{\partial^{2}}{\partial x^{2}}+V_{1}-i \hbar \frac{\partial}{\partial t}\right) \psi_{1}(x, t)+V_{12} \psi_{2}(x, t)=0, \\
& \left(-\frac{\hbar^{2}}{2 m} \frac{\partial^{2}}{\partial x^{2}}+V_{2}-i \hbar \frac{\partial}{\partial t}\right) \psi_{2}(x, t)+V_{12} \psi_{1}(x, t)=0 .
\end{aligned}
$$

Throughout the paper the subscript refers to the two surfaces $V_{1}$ and $V_{2}$.

Generalization of the single-surface quantum trajectory description summarized by Eqs. (1)-(4) is based on the substitution of the wave function in terms of its real amplitude and phase into Eq. (15),

$$
\psi_{i}(x, t)=A_{i}(x, t) \exp \left(\frac{i}{\hbar} S_{i}(x, t)\right), \quad i=1,2,
$$

followed by the separation of real and imaginary parts. This Bohmian formulation was presented and implemented using the exact quantum trajectories by Wyatt et al. [50]. The polar representation of $\psi_{i}$ also underlies a widely used surface hopping method [51] characterized by purely classical dynamics of trajectories "jumping" between the surfaces, and the quantum-classical mixing approaches of Refs. [52-54]. Other methods of nonadiabatic dynamics based on classical or semiclassical mechanics are discussed in Ref. [55].

We start developing the semiclassical trajectory description by combining the Bohmian nonadiabatic generalization with the concept of trajectory weights. Using the wavefunction densities $\rho_{i}(x, t)=A_{i}^{2}(x, t)$ and identifying $p_{i}$ $=S_{i}^{\prime}(x, t)$, in the moving frames of reference defined by appropriate momenta $p_{i}$ [Eq. (5)], the real parts of Eqs. (15) give the time evolution of the action function $S_{i}\left(x_{i}, t\right)$,

$$
\frac{d S_{i}\left(x_{i}, t\right)}{d t}=\frac{p_{i}^{2}}{2 m}-\left(V_{i}+U_{i}+U_{i j}\right),
$$

where $i=1,2, j=1,2$, and $j \neq i$. $U_{i}$ is the single-surface quantum potential given by Eq. (2). $U_{i j}$ is an additional potential due to coupling,

$$
\begin{gathered}
U_{i j}=V_{12} \rho_{j i} \cos (\Delta S), \\
\rho_{j i}=\sqrt{\frac{\rho_{j}(x, t)}{\rho_{i}(x, t)}}, \quad \Delta S=\frac{1}{\hbar}\left[S_{1}(x, t)-S_{2}(x, t)\right] .
\end{gathered}
$$

The imaginary parts of Eqs. (15) give the following expression for the trajectory weights:

$$
\frac{d w_{i}}{d t}=-2 V_{12} \rho_{j i} \sin (\Delta S) w_{i} .
$$

In this formulation there are two sets of trajectories, one on each surface, evolving in time. Coupling affects both the dynamics of trajectories and the time evolution of their weights. The trajectory propagation can be accomplished using the AQP method, for example using $U_{i}$ defined by Eq. (7), which can describe the quantum behavior of nuclei on surfaces. In the region of space where $V_{12}$ is appreciable, two additional quantities $\Delta S$ and $\rho_{j i}$ have to be evaluated along the trajectories on each surface. Low- (quadratic-)order fitting of the phase $S_{i}$ with a weighting function $V_{12} w_{i}$ is cheap and sufficiently accurate for localized $V_{12}$. The quantity $\rho_{j i}$ is estimated using the AQP parameters found from Eq. (6) defining the single-surface potentials $U_{i}$. Alternatively, it can be determined by approximating each density as a Gaussian from the first and second moments of the trajectory distribution using $V_{12} w_{i}$ as a weighting function. The latter strategy is consistent with the fitting of $S_{i}$. Overall, this approach is nearly as efficient as the single-surface AQP method, and might be adequate when the trajectory dynamics is smooth, such as for asymptotically degenerate $V_{1}$ and $V_{2}$ coupled by a localized $V_{12}[50]$.

Though formally exact, the formulation of Eqs. (17)-(20) has certain drawbacks. $U_{i j}$ given by Eq. (18) involves a pos- 
sibly singular ratio of the densities. For instance, a typical initial condition for a multisurface problem is a single populated state, such as $\rho_{2}(x, 0)=0$. In Ref. [50] this problem was circumvented by propagating two sets of initial wave packets with nonzero population on both surfaces, whose linear combination gives the desired initial condition. Alternatively, the singularity can be canceled at $t=0$ by the choice of initial conditions for the trajectories. If $\rho_{2}(x, 0)=0$, one can set up trajectories on the second surface with the same initial positions and momenta as on the lower surface, and introduce a phase shift between $\psi_{1}(x, 0)$ and $\psi\left(x_{2}, 0\right), \Delta S(x, 0)= \pm \pi / 2$, so that the singularity in $U_{21}$ cancels at $t=0$ and the subsequent time evolution is stable. The sign of the phase shift depends on the derivative of $V_{2}$.

In the context of semiclassical dynamics there is also a conceptual problem with the Bohmian formulationnonadiabatic behavior is an intrinsically quantum effect that does not vanish in the semiclassical limit. In practice, this is manifested through the behavior of the coupling terms in Eqs. (17) and (20) as $\hbar \rightarrow 0$. The force on trajectories derived from $U_{i j}$ has a contribution proportional to $\cos ^{\prime}(\Delta S)$ $=\hbar^{-1}\left[p_{2}(x)-p_{1}(x)\right] \sin (\Delta S)$, which does not go to zero in this limit and, even worse, becomes large and oscillatory because of the $\hbar^{-1}$ dependence. Apart from computational challenges, this asymptotic behavior shows that the Bohmian formulation is incompatible with a semiclassical method, where propagation is expected to become classical as $\hbar \rightarrow 0$.

We applied the Bohmian formulation to the curvecrossing model system [14] discussed in detail in Sec. IV. We found that, in general, the dynamics and time dependence of the trajectory weights were unstable due to large forces coming from the coupling terms. For high-energy wave packets propagation was stable and accurate long enough to compute transmission probabilities. It may be possible to stabilize propagation of lower-energy wave packets by imposing cutoffs on the forces or by other numerical solutions, but we do not pursue this approach here. Instead, we use the mixed wave-function representation which better captures the physics of nonadiabatic processes.

\section{B. The mixed representation approach}

Analysis of the Bohmian nonadiabatic formulation suggests that a practical semiclassical treatment of nonadiabatic dynamic systems should combine semiclassical dynamics (with the proper $\hbar \rightarrow 0$ limit) of wave functions with quantum transitions between the surfaces. To achieve this we use a mixed polar-coordinate space wave-function representation: the polar part will give a compact representation of the overall dynamics of the wave function, whereas the coordinate space part will describe generally complex "population" amplitudes. Ideally, all functions are smooth compared to the wave function in coordinate representation.

The components of the wave function are represented as products,

$$
\psi_{i}(x, t)=\phi_{i}(x, t) \chi_{i}(x, t), \quad i=1,2 .
$$

The result of substituting Eq. (21) into Eq. (15) can be separated into two parts, defining the time dependence of $\phi_{i}(x, t)$ and $\chi_{i}(x, t)$. This separation is not unique: we choose it so that $\phi_{i}(x, t)$ satisfies the single-surface $\mathrm{SE}$

$$
i \hbar \frac{\partial}{\partial t} \phi_{i}(x, t)=-\frac{\hbar^{2}}{2 m} \frac{\partial^{2}}{\partial x^{2}} \phi_{i}(x, t)+V_{i} \phi_{i}(x, t) .
$$

Representing $\phi_{i}(x, t)$ in polar form, $\phi_{i}(x, t)$ $=a_{i}(x, t) \exp \left[i s_{i}(x, t) / \hbar\right]$, and solving Eq. (22) in terms of trajectories as given by Eq. (4) with $p_{i}=s_{i}^{\prime}(x, t)$, the remaining terms of Eq. (15) give the following time dependence of $\chi_{i}(x, t)$ in the moving frame of reference:

$$
i \hbar \frac{d}{d t} \chi_{i}\left(x_{i}, t\right)=K_{i}+V_{12} \frac{\phi_{j}\left(x_{i}, t\right)}{\phi_{i}\left(x_{i}, t\right)} \chi_{j}\left(x_{i}, t\right) .
$$

$K_{i}$ contains spatial derivatives of $\chi_{i}\left(x_{i}, t\right)$,

$$
K_{i}=-\frac{\hbar^{2}}{2 m}\left(\chi_{i}^{\prime \prime}\left(x_{i}, t\right)+2 \frac{a_{i}^{\prime}\left(x_{i}, t\right)}{a_{i}\left(x_{i}, t\right)} \chi_{i}^{\prime}\left(x_{i}, t\right)\right) .
$$

The time propagation of $\phi_{i}(x, t)$ can be accomplished semiclassically using the AQP method. For an efficient solution of Eq. (23) we need a small basis set of functions to evaluate $\chi_{i}^{\prime}\left(x_{i}, t\right), \chi_{i}^{\prime \prime}\left(x_{i}, t\right)$, and $\chi_{j}\left(x_{i}, t\right)$. The coefficients of the expansion can be found as the standard least squares fit procedure [56]. The functions $\phi_{i}(x, t)$ can be approximated with a Gaussian form in the interaction region from the moments of the trajectory distribution. The approximate terms $a_{i}^{\prime}\left(x_{i}, t\right) / a_{i}\left(x_{i}, t\right)$ are already available from the AQP procedure given by Eqs. (6) and (7).

We emphasize that $\chi_{i}\left(x_{i}, t\right)$ evolves in time along trajectories according to Eq. (23) and is not solved for in the basis set representation. The basis set is needed for evaluation of the right-hand side of Eq. (23). In particular, the term $K_{i}$ is of $\hbar^{2} / m$ order and is expected to be small or even negligible in the semiclassical regime, while the function $\chi_{i}\left(x_{i}, t\right)$ itself may not be small. In fact, it cannot be small everywhere, since the total wave function remains normalized. The most efficient way to evaluate the coupling term in Eq. (23) is to approximate $\chi_{j}\left(x_{i}, t\right)$ globally, such as by using a small set of functions. Nevertheless, more accurate and more expensive local approximation schemes might also be acceptable, especially for a localized coupling potential, when the coupling term is nonzero only for a limited time interval along a given trajectory.

We choose a particular form for a small basis suitable for evaluation of the right-hand side in Eq. (23) by analyzing the limiting form of this equation. Consider a system in the highenergy limit with initially identical $\phi_{i}(x, 0)$, but different $\chi_{i}(x, 0)$. In this regime the time evolution of $\phi_{i}(x, t)$ is essentially a free-space propagation and the initial phases can be adjusted so that $\phi_{1}(x, t) / \phi_{2}(x, t) \approx 1$, while the wave packets quickly pass through the interaction region. The terms $K_{i}$ are on the order of $\hbar^{2} / m$ and can be omitted. Then, Eq. (23) in the stationary frame of reference can be rewritten as

$$
\frac{\partial \chi_{i}(x, t)}{\partial t}+\frac{i}{\hbar} V_{12}\left(x-q_{t}\right) \chi_{j}(x, t)=0,
$$

where $\left\{q_{t}, p\right\}$ are the position and momentum of a trajectory (the same on all surfaces in the given limit) at time $t$. These 
equations have a general solution involving an integral of the coupling along a trajectory $I(x, t)=m(\hbar p)^{-1} \int V_{12}\left(x-q_{t}\right) d q_{t}$,

$$
\chi_{i}(x, t)=(-1)^{i} f_{1}(x) e^{-i I(x, t)}+f_{2}(x) e^{i I(x, t)} .
$$

Therefore, for localized $V_{12}$, the functions $\left\{1, V_{12}, \int V_{12} d x\right\}$ and the same functions multiplied by $x$ to account for asymmetry might be adequate to serve as a small basis set for $\chi_{i}(x, t)$.

The mixed representation formulation with a small basis for $\chi_{i}(x, t)$ is expected to be accurate for systems with a localized interaction region in the semiclassical regime, when the terms $K_{i}$ in Eq. (23) are small and the time of interaction between the surfaces is short. The nonadiabatic effect can be large even in this regime if the coupling between the surfaces is strong, which is the case in the example below.

\section{RESULTS AND DISCUSION}

\section{A. Semiclassical energy-resolved probabilities}

We applied the mixed representation approach to the curve-crossing model problem of Tully [14], which is widely used as a test in nonadiabatic dynamics. Atomic units except for the unit of energy are used below. The potentials and coupling,

$$
\begin{aligned}
& V_{1}(x)= \begin{cases}\frac{V^{*}}{2} \exp (a x), & x \leqslant 0, \\
V^{*}-\frac{V^{*}}{2} \exp (-a x), & x>0,\end{cases} \\
& V_{2}(x)=V_{1}(-x), \quad V_{12}=V_{c} \exp \left(-z x^{2}\right),
\end{aligned}
$$

with parameter values $a=1.6 \mathrm{bohr}^{-1}, \quad z=1.0 \mathrm{bohr}, \quad V^{*}$ $=52.5 \mathrm{~kJ} / \mathrm{mol}$, and $V_{c}=V^{*} / 4$, are shown on Fig. 1. The mass of the particle is $m=2000$. The wave-packet correlation function formulation of scattering theory [57] is used to obtain the energy-resolved reaction probability from the reactant region of $V_{1}$ to the product region of the surfaces $V_{1}$ or $V_{2}$. A wave function $\psi_{1}(x, t)$ initially localized in the asymptotic region of the surface $V_{1}$ is given by Eq. (21) with $\chi_{1}(x, 0)$ $=1$ and

$$
\phi_{1}(x, 0)=\left(\frac{2 \alpha}{\pi}\right)^{1 / 4} \exp \left[-\alpha\left(x-x_{0}\right)^{2}+i p_{0}\left(x-x_{0}\right)\right] .
$$

Initially, the population on the surface $V_{2}$ is zero, which in the mixed representation is equivalent to $\chi_{2}(x, 0)=0$. Formally, $\phi_{2}(x, 0)$ can be an arbitrary normalizable function. In practice a $\phi_{2}(x, t)$ that has large overlap with $\phi_{1}(x, t)$ in the interaction region is preferred. Such a choice will give smoother $\chi_{1}(x, t)$ and $\chi_{2}(x, t)$, since the changes in $\chi_{2}(x, t)$ will describe the transitions. Otherwise, in order to produce finite values of the wave function $\psi_{2}(x, t)$ in the interaction region, $\chi_{2}(x, t)$ will have to be large to compensate for negligible weights of the trajectories of the second surface in this region. The choice $\phi_{2}(x, 0)=\phi_{1}(x, 0)$ works for the given system, since $V_{1}$ and $V_{2}$ differ from a constant in the same region of space as $V_{12}$.
The central quantity of interest in the correlation function formulation is the overlap $C_{i}(t)$ of the evolving wave function with the stationary wave packet $\psi_{0}(x, 0)$,

$$
C_{i}(t)=\left\langle\psi_{i}(x, t)\right| \psi_{0}(x, 0) .
$$

$\psi_{0}(x, 0)$ representing the product channel (see Fig. 1) is chosen here as $\psi_{0}(x, 0)=(2 \alpha / \pi)^{1 / 4} \exp \left[-\alpha\left(x+x_{0}\right)^{2}+i p_{0}\left(x+x_{0}\right)\right]$. Fourier analysis of $C_{1}(t)$ gives the probability $P_{11}(E)$ for the reaction on the surface $V_{1}$-no intersurface transition in the diabatic representation, while $C_{2}(t)$ gives the probability $P_{12}(E)$ for the reaction with the transition to the second surface $V_{2}$,

$$
P_{1 i}(E)=\left|S_{1 i}(E)\right|^{2}=\eta_{2}(E) \eta_{i}(E)\left|F_{i}(E)\right|^{2} .
$$

$F_{i}(E)$ is the Fourier transform of the correlation function,

$$
F_{i}(E)=\int C_{i}(t) e^{i E t} d t, \quad i=1,2 .
$$

$S_{11}(E)$ and $S_{12}(E)$ are elements of the scattering matrix for the reaction on surface $V_{1}$ and with the transition to surface $V_{2}$, respectively. The functions $\eta_{i}(E)$ normalize the initial reactant and product wave packets, which are superpositions of the energy eigenstates, with respect to energy. For purely incoming $\psi_{1}(x, 0)$ and purely outgoing $\psi_{0}(x, 0)$ the normalization is

$$
\begin{aligned}
& \eta_{i}(E)=\exp \left(\frac{\left(p_{i}-p_{0}\right)^{2}}{2 \alpha}\right) \sqrt{\frac{\alpha}{2 \pi}} \frac{p_{i}}{m}, \\
& p_{1}=\sqrt{2 m\left(E-V^{*}\right)}, \quad p_{2}=\sqrt{2 m E} .
\end{aligned}
$$

$V^{*}$ is the difference in the potential in the reactant and product regions of the surface $V_{1}$. A more general form of wave packets is discussed in Ref. [58]. In the high-energy regime, there is no reflection, and the two reaction probabilities add up to $1, P_{12}(E)+P_{11}(E)=1$.

A typical behavior of $\chi_{1}(x, t)$ and $\chi_{2}(x, t)$ solving Eq. (25) is shown on Fig. 2. The time evolution of the center of the wave packet was $q_{t}=x_{0}+p_{0} t / m$ with $x_{0}=-2$ and $p_{0}=45$ with the potential $V_{12}$ defined in Eq. (27). The functions are shown at time increments of $T=50$ a.u. beginning at $t=T$. The coupling term is a product $V_{12} \chi_{i}(x, t)$, so $\chi_{i}(x, t)$ is needed only in the interaction region and the suggested basis is adequate. This basis also gives reasonable estimates of $K_{i}$ terms, which should be small in the asymptotic regions, at least in the semiclassical regime of large $m$ : nonadiabatic transitions do not occur outside the interaction region and $K_{i}$ is small.

The scattering matrix amplitudes $\left|S_{1 i}(E)\right|$ are shown on Fig. 3. The entire range of energy is covered by two wave packets. The high-energy wave packet defined by Eq. (28) with parameter values $\left\{\alpha=12, x_{0}=-2.35, p_{0}=25\right\}$ was represented by 145 trajectories propagated up to $t=1000$ a.u. This wave packet has contributions from the energy eigenstates in the range of $E=[200,700] \mathrm{kJ} / \mathrm{mol}$. The low-energy wave packet specified by $\left\{\alpha=12, x_{0}=-2.35, p_{0}=12\right\}$, was propagated up to $t=3000$ a.u. Computation of the long-time correlation function in this case required about 700 trajectories. 


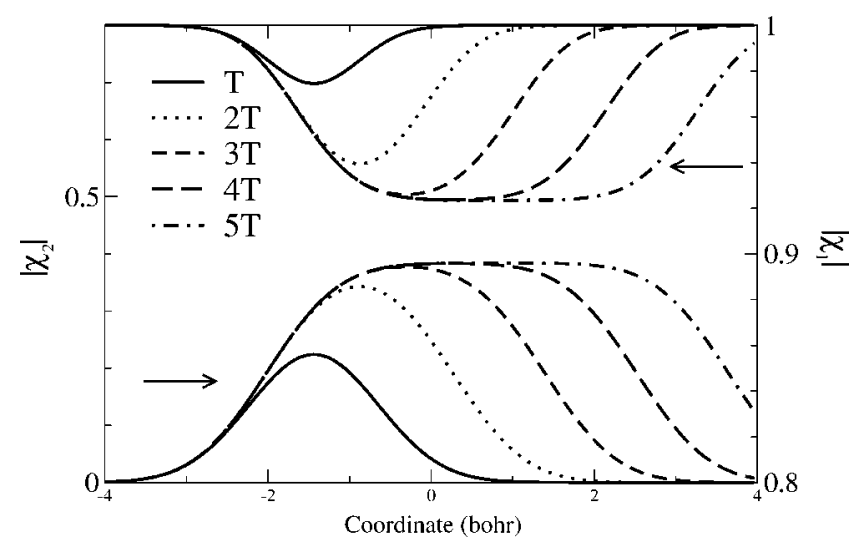

FIG. 2. A typical high-energy solution for the population functions $\chi_{1}(x, t)$ and $\chi_{2}(x, t)$ with the initial conditions $\chi_{1}(x, 0)=1$ and $\chi_{2}(x, 0)=0$ shown for the system of Eq. (27) at five times, $t$ $=T, \ldots, 5 T$. $(T=50$ a.u. See text for details. $)$ The scales for $\chi_{1}$ and $\chi_{2}$ are different and are shown on the right and left vertical axes, respectively.

The initial positions of the trajectories sampled $\phi_{i}(x, 0)$ uniformly. Only trajectories with weights greater than $10^{-8}$ were propagated. A nonuniform sampling would considerably reduce the number of trajectories for the low-energy wave packet, since the AQP parameters converge with fewer trajectories than $C_{i}(t)$ itself. Each wave-packet calculation gives probabilities for about 40 energy values. For comparison, the surface hopping results in Ref. [14] were obtained with 5000 trajectories for each energy value.

Reaction on the surface $V_{1}$ occurs only for energies above $V^{*}$, while nonadiabatic reaction can proceed for all positive energies. Scattering matrix amplitudes for both reactions are shown on Fig. 3(c). (Not all points are shown.) They are compared to the quantum results obtained with the usual split-operator method [59]. The agreement at high energies is excellent. Semiclassical probabilities for energies close to and below $V^{*}$ lose accuracy, though they exhibit features somewhat similar to the quantum result. At low energies the behavior of the system is influenced by the resonances which are not fully reproduced by the semiclassical method. The behavior of the wave-packet correlation functions supports this conclusion. The function $C_{1}(t)$ for the low-energy wave packet, shown on Fig. 3(a), agrees with the quantum result quite well for up to $t=1000$. It develops a slight lag in the phase for later times, corresponding to the low-energy components with $E$ close to $V^{*}$. The energy components below $V^{*}$ contribute only to the nonadiabatic process. The corresponding correlation function $C_{2}(t)$ shown on panel (b) has a lower amplitude at long times compared to the quantum result, in addition to the phase lag. Inaccuracy in the AQP is also largest in the lower-energy regime.

The mixed representation approach as described above does not conserve wave function normalization, which in discretized form is

$$
N=\sum_{k} w_{1}^{k}\left|\chi_{1}\left(x_{1}^{k}, t\right)\right|^{2}+\sum_{l} w_{2}^{l}\left|\chi_{2}\left(x_{2}^{l}, t\right)\right|^{2}
$$

where $k$ and $l$ label the trajectories. [A straightforward way of imposing conservation of the norm is to include it as the
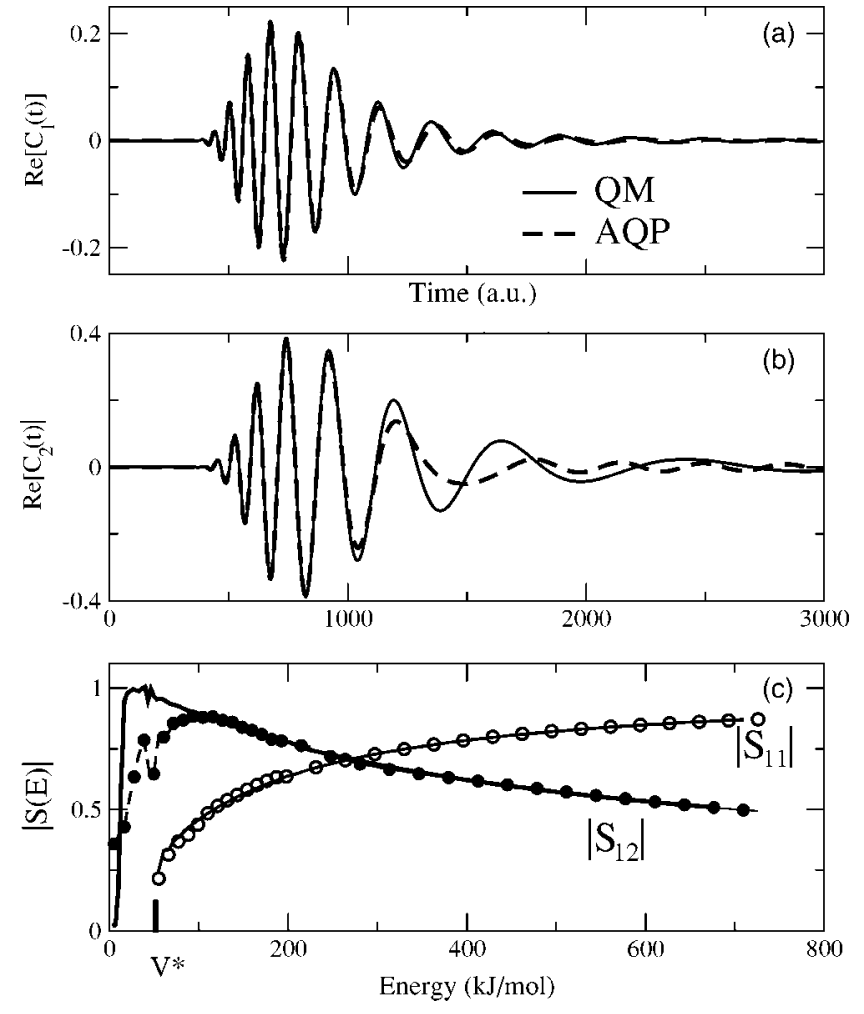

FIG. 3. Dynamics and reaction probabilities. (a) The real parts of the quantum (solid line) and AQP (dash line) correlation functions for the reaction on the surface $V_{1}$. (b) The same as (a) for the nonadiabatic reaction. (c) The energy-resolved scattering matrix amplitudes for the diabatic, $\left|S_{11}\right|$, and for the nonadiabatic, $\left|S_{12}\right|$, processes: the quantum result is shown with thin and thick solid lines for the two processes, respectively; the corresponding AQP results are shown with open and filled circles. $V^{*}$ marks the threshold of the diabatic reaction.

Lagrange multiplier at the stage of expanding $\chi_{i}(x, t)$.] In these calculations the maximum deviation of $N$ from unity was $4 \%$ and $0.1 \%$ for the low- and high-energy wave packets, respectively. Overall, the quality of the semiclassical results is surprisingly good, especially considering the fact that the energy-resolved probabilities are much more sensitive to the details of dynamics than the wave packet probabilities, where cancellation of errors may occur due to averaging over the density.

\section{B. Effect of the quantum terms on dynamics}

The trajectory description of nonadiabatic dynamics allows us to examine effects of the quantum terms influencing dynamics - the single-surface quantum potential $U_{i}$ and the kinetic energy term $K_{i}$ in Eq. (23). Figure 4 shows the singlesurface probabilities for the high-energy wave packet when the $K_{i}$ terms were set to zero (calculation A) and when the quantum potential was set to zero as well (calculation B). Panel (a) shows just the Fourier transform of the correlation functions, $\left|F_{1}(E)\right|$, given by Eq. (31) for calculations A and B and for the full semiclassical calculation, which in this case reproduces the exact quantum result. 

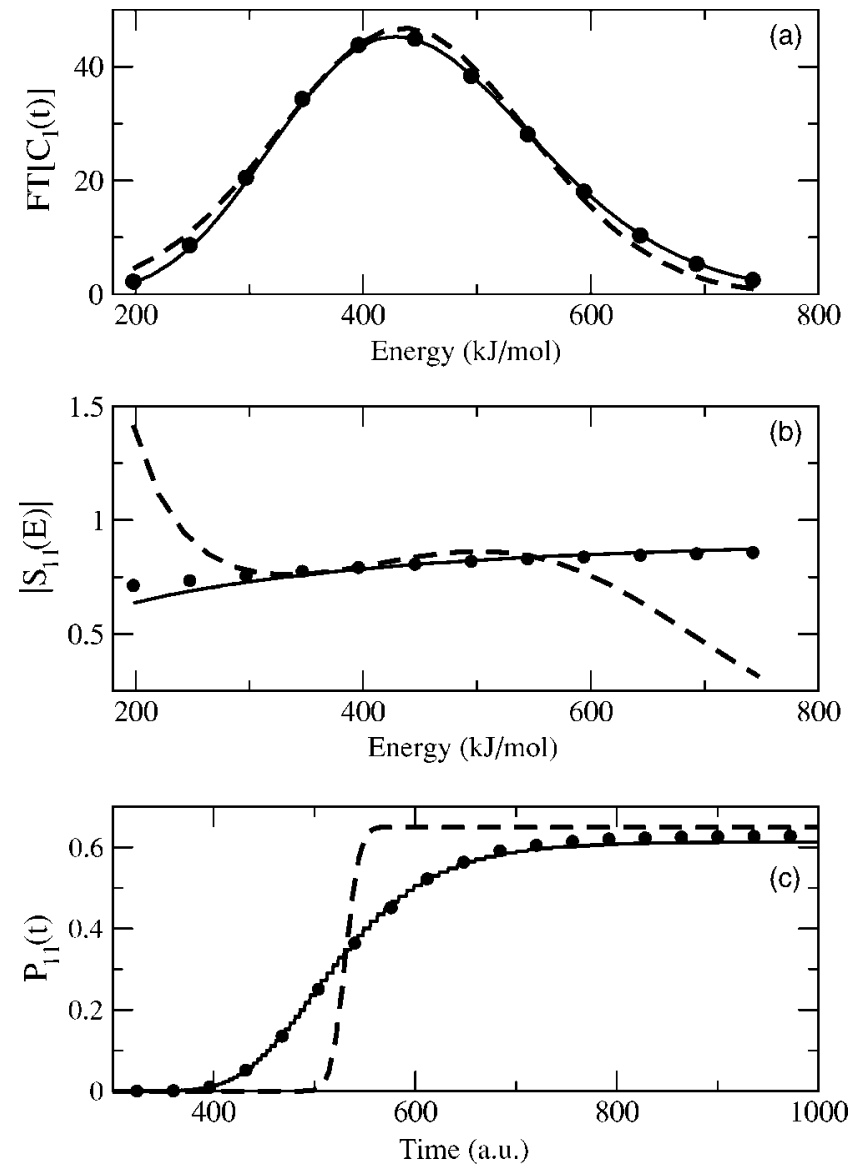

FIG. 4. Effect of the quantum terms for the diabatic reaction. (a) The Fourier transform of the correlation function: solid line, circles, and dashed line show, respectively, the full AQP result and the results with the kinetic energy $K_{i}$ omitted (calculation A) and with the quantum potential $U_{i}$ and $K_{i}$ omitted (calculation B). (b) The energy-resolved scattering matrix amplitude. (c) The wave-packet reaction probability as a function of time. The legend in (b) and (c) is the same as in (a).

The Fourier transforms for the calculation B are somewhat shifted, whereas the difference between the calculation A and the full semiclassical calculation is barely visible on the plot. However, these small discrepancies become much more noticeable once the energy normalization is included. The panel (b) shows the energy-resolved scattering matrix amplitudes $\left|S_{11}(E)\right|$. The results of the calculation A are in reasonable agreement with the exact result, though there is some difference in the slope of the two curves. The results of the calculation B agree with the exact result for the energy corresponding to the average wave-packet energy, whereas the probability for other energies shows large discrepancy, including the nonphysical result at low energies. Such behavior is easily explained by the role of the quantum potential in this formalism. For a chosen wave packet all trajectories have the same initial momentum $p_{0}$. The quantum force redistributes the energy of the quantum potential among the trajectories in the course of dynamics. This effect, missing in the calculation $\mathrm{B}$, has a big influence on the energy-resolved results.
The effect of the quantum potential is less dramatic on the wave-packet reaction probability computed as

$$
P_{11}(t)=\sum_{k^{\prime}} w_{1}\left(x_{1}^{k}, t\right)\left|\chi_{1}\left(x_{1}^{k}, t\right)\right|^{2}
$$

with just the reactive trajectories, $x_{1}^{k}>4$, included in the sum. $P_{11}(t)$ is shown on panel (c). The effect of $K_{i}$ terms is rather small: the long time probability is off by 0.01 and the time dependence of $P_{11}(t)$ in this case closely follows the exact result. In case of the omitted quantum potential, $P_{11}(t)$ looks like a step function-all trajectories cross the line $x=4$ at about the same time. The final probability is off by only 0.04 from the exact result, but the discrepancy can be much worse in more than one dimension. In one dimension the probability is largely defined by the initial momentum and not by the details of the dynamics. Also note that the effect of the quantum potential strongly depends on the parameters of the wave packet. It should be much smaller for the wide (small $\alpha$ ), essentially monoenergetic wave packets used in Ref. [14].

\section{A case of total nonadiabatic transition}

We expect the mixed representation approach to be accurate for high energies, because in this regime the AQP is more accurate, and because the form of the basis set is derived in the high-energy limit. We did not make explicit assumptions about the coupling strength, though weaker coupling should be better described by the semiclassical method. We examined the effect of the coupling strength by performing additional calculations with the coupling parameter $V_{c}$ twice smaller and twice larger than the original value $V_{c}$ $=V^{*} / 4$. It was found that the quality of the results for the small coupling is similar to those presented on Fig. 3, except that accuracy of $P_{12}(E)$ deteriorates at lower energies, $E$ $<70 \mathrm{~kJ} / \mathrm{mol}$, compared to $E<100 \mathrm{~kJ} / \mathrm{mol}$ obtained for the original coupling strength.

In case of the increased coupling we found the quality of semiclassical result also changed according to expectations. The accuracy "threshold" energy for the strong coupling was $E=145 \mathrm{~kJ} / \mathrm{mol}$. Interestingly enough there is a point of total nonadiabatic transmission at $E \approx 247.5 \mathrm{~kJ} / \mathrm{mol}$. In the course of dynamics the wave-function amplitude, initially localized on the surface $V_{1}$, is quickly transferred to the second surface developing a node in $\left|\psi_{1}(x, t)\right|$ as it passes through the interaction region. This feature is reflected in $C_{1}(t)$ whose absolute value has two local maxima. Figure 5(a) shows two snapshots of the wave-function amplitude at times $t=300$ and 400 for the wave packet given by Eq. (28) with parameters $\left\{\alpha=12, x_{0}=-2.35, p_{0}=22\right\}$. This feature is well reproduced in the semiclassical calculation performed with 145 trajectories. Dynamics in the purely Bohmian formulation described in Sec. III A would be very unstable for this system, due to the node in $\left|\psi_{1}(x, t)\right|$. The semiclassical scattering matrix amplitude shown on panel (b) has a minimum of 0.015 at the correct energy within the resolution of the spectrum and is in good overall agreement with the quantum result. 

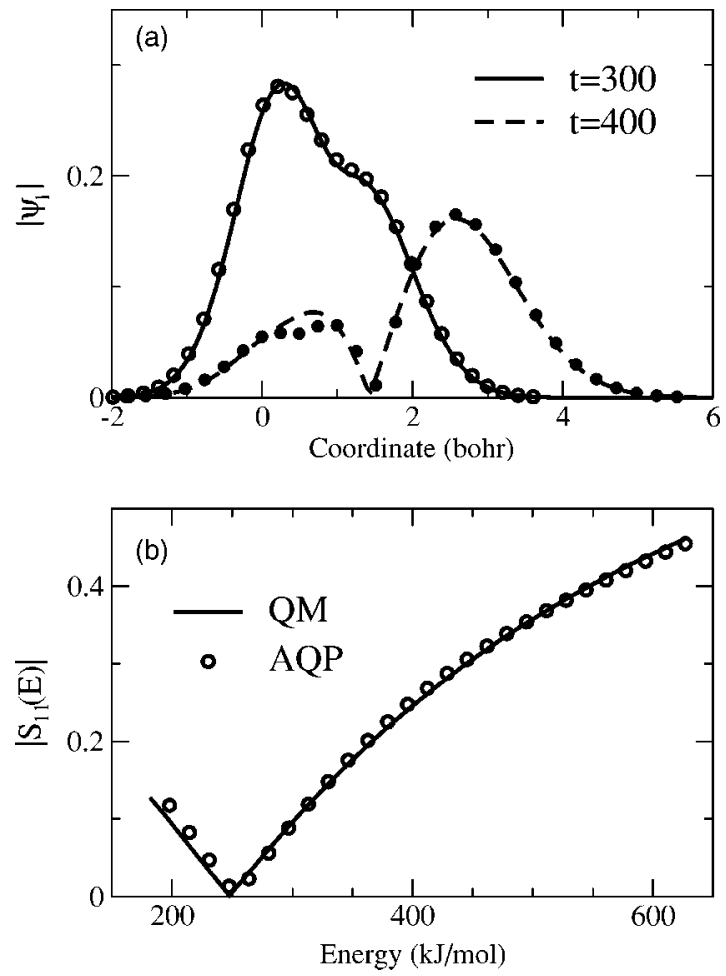

FIG. 5. The system with increased coupling $V_{c}=V^{*} / 2$. (a) The wave-function amplitude $\left|\psi_{1}(x, t)\right|$, as the wave packet passes through the interaction region at $t=300$ and 400: the quantum results are shown with solid and dashed lines for the two times, respectively; the corresponding AQP results are shown with open and filled circles. (b) The quantum (solid line) and the AQP (circles) scattering matrix amplitudes for the diabatic process.

\section{CONCLUSIONS}

We have extended the semiclassical wave-packet propagation approach based on the approximate quantum potential to nonadiabatic dynamics. The original hydrodynamic or de Broglie-Bohm formulation can be generalized to the case of multiple coupled electronic potential surfaces within the approximate quantum potential approach. However, this formulation is inconsistent with the concept of semiclassical dynamics, defined as being systematically improvable toward QM dynamics in the $m \rightarrow \infty$ limit for general wave functions. Therefore, we use a mixed wave-function representation, which gives a compact description of the wave-packet dynamics in terms of modified quantum trajectories with the correct semiclassical limit of the vanishing single-surface quantum potential. The intrinsically quantum effect of transitions between potential surfaces is described in coordinate space. An additional quantity - the population amplitude-is associated with each trajectory. Changes in time in this function represent transitions. Moreover, the function itself has the flexibility to describe zero-wave-function densities in the initial conditions or as they develop in the course of the dynamics, without dealing with the singularities typical for the Bohmian formulation. In the high-energy regime, for lo- calized coupling the time evolution of the population amplitude can be efficiently accomplished using a small basis related to a given coupling potential.

The mixed representation approach is applied to the widely used curve-crossing model of Tully [14]. The correlation function formulation of scattering theory [57] is used to compute the energy-resolved probabilities of the diabatic and nonadiabatic reactions. This formulation enables us to use highly localized initial wave functions located close to the coupling region. This setup requires a shorter propagation time, which, generally, improves the accuracy of approximate methods. The energy-normalization procedure, on the other hand, is very sensitive to the quality of the correlation functions, since it does not benefit from the error cancellation due to averaging over energies. Our semiclassical calculations yield probabilities in the range of energy up to $700 \mathrm{~mJ} / \mathrm{mol}$ from just two wave-packet propagations using 700 (low-energy wave-packet) and 145 (high-energy wavepacket) trajectories. The low-energy probabilities of the nonadiabatic reaction influenced by resonances are underestimated. The probabilities for high energies, $E>100$ $\mathrm{kJ} / \mathrm{mol}$, are in excellent agreement with the quantum results. Moreover, the mixed representation approach allows us to analyze the relative importance of the quantum terms for a given system. We find that for localized wave packets the quantum potential $U_{i}$ drastically affects the reaction probabilities. The influence of the kinetic energy of the population amplitude, $K_{i}$, which is an $\bar{\hbar}^{2} / m$-order term, is much smaller, and, probably, can be omitted if just the expectation values are of interest. We have tested a range of coupling strength and observed a high-energy point of the total nonadiabatic transition at increased coupling. This behavior is accurately reproduced by our semiclassical method.

Further studies and applications are needed for a more complete assessment of the mixed representation approach to semiclassical nonadiabatic dynamics. Future applications should include time-dependent coupling induced, for example, by ultrashort laser pulses [60] and multiple or multidimensional potential surfaces. The description of the lowenergy regime can be improved at the expense of using larger basis sets, or local interpolation procedures. Systems with nonlocalized coupling can be treated by the same approach, in principle. Using a single set of quantum trajectories-a single polar part for all components of a wave function-is an intriguing possibility for systems with multiple "parallel" surfaces. For the present, we have demonstrated that nonadiabatic dynamics based on semiclassical propagation with the approximate quantum potential and the quantum description of transitions can be efficient and accurate in the semiclassical regime.

\section{ACKNOWLEDGMENTS}

Acknowledgment is made to the Donors of the American Chemical Society Petroleum Research Fund, for support of this research. This work was also supported by the NSF/ EPSCOR under Grant No. EPS-0296165. 
[1] C. Dekker and M. A. Ratner, Phys. World 14 (8), 29 (2001).

[2] J. D. Lear, Z. R. Wasserman, and W. F. DeGrado, Science 240, 1177 (1988).

[3] Y. Cha, C. J. Murray, and J. P. Klinman, Science 243, 1325 (1989).

[4] H. Lee and J. C. Light, J. Chem. Phys. 120, 5859 (2004).

[5] L. J. Butler, Annu. Rev. Phys. Chem. 49, 125 (1998).

[6] L. Sun, K. Song, and W. L. Hase, Science 296, 875 (2002).

[7] S. C. Ammal, H. Yamataka, M. Aida, and M. Dupuis, Science 299, 1555 (2003).

[8] Electron Transfer in Inorganic, Organic and Biological Systems, edited by J. R. Bolton, N. Mataga, and G. McLendon, (American Chemical Society, Washington, DC, 1991).

[9] R. Engleman, Non-Radiative Decay of Ions and Molecules in Solids (Norh-Holland, Amsterdam, 1979).

[10] R. J. Gordon and S. A. Rice, Annu. Rev. Phys. Chem. 48, 601 (1997).

[11] D. H. Zhang and J. Z. H. Zhang, J. Chem. Phys. 101, 1146 (1994).

[12] D. H. Zhang and J. Z. H. Zhang, J. Chem. Phys. 103, 6512 (1995).

[13] F. Huarte-Larranga and U. Manthe, J. Phys. Chem. A 105, 2522 (2001).

[14] J. C. Tully, Faraday Discuss. 110, 407 (1998).

[15] M. F. Herman and E. Kluk, Chem. Phys. 91, 27 (1984).

[16] K. G. Kay, J. Chem. Phys. 100, 4377 (1994).

[17] W. H. Miller, J. Phys. Chem. A 105, 2942 (2001).

[18] V. Guallar, V. S. Batista, and W. H. Miller, J. Chem. Phys. 110, 9922 (1999).

[19] V. Guallar, B. F. Gherman, and W. H. Miller, J. Am. Chem. Soc. 124, 3377 (2002).

[20] D. Bohm, Phys. Rev. 85, 166 (1952).

[21] A. S. Sanz, F. Borondo, and S. Miret-Artes, J. Phys.: Condens. Matter 14, 6109 (2002).

[22] I. Burghardt and L. S. Cederbaum, J. Chem. Phys. 115, 10303 (2001).

[23] I. Burghardt and L. S. Cederbaum, J. Chem. Phys. 115, 10312 (2001).

[24] I. Burghardt and K. B. Moller, J. Chem. Phys. 117, 7409 (2002).

[25] J. B. Maddox and E. R. Bittner, J. Phys. Chem. B 106, 7981 (2002).

[26] E. R. Bittner, J. B. Maddox, and I. Burghardt, Int. J. Quantum Chem. 89, 313 (2002).

[27] A. Donoso and C. C. Martens, Phys. Rev. Lett. 87, 223202 (2001).

[28] C. J. Trahan and R. E. Wyatt, J. Chem. Phys. 119, 7017 (2003).

[29] B. K. Dey, A. Askar, and H. Rabitz, J. Chem. Phys. 109, 8770 (1998).

[30] R. E. Wyatt and K. Na, Phys. Rev. E 65, 016702 (2002).
[31] E. R. Bittner, J. Chem. Phys. 112, 9703 (2000).

[32] R. E. Wyatt and E. R. Bittner, J. Chem. Phys. 113, 8898 (2000).

[33] B. K. Kendrick, J. Chem. Phys. 119, 5805 (2003).

[34] D. Babyuk and R. E. Wyatt, J. Chem. Phys. 121, 9230 (2004).

[35] B. Poirier, J. Chem. Phys. 121, 4501 (2004).

[36] C. J. Trahan, K. Hughes, and R. E. Wyatt, J. Chem. Phys. 118, 9911 (2003).

[37] J. B. Maddox and E. R. Bittner, J. Chem. Phys. 119, 6465 (2003).

[38] J. Liu and N. Makri, J. Phys. Chem. A 108, 5408 (2004).

[39] S. Garashchuk and V. A. Rassolov, J. Chem. Phys. 118, 2482 (2003).

[40] S. Garashchuk and V. A. Rassolov, Chem. Phys. Lett. 376, 358 (2003).

[41] S. Garashchuk and V. A. Rassolov, J. Chem. Phys. 120, 1181 (2004).

[42] V. A. Rassolov and S. Garashchuk, J. Chem. Phys. 120, 6815 (2004).

[43] S. Garashchuk and V. A. Rassolov, Chem. Phys. Lett. 364, 562 (2002).

[44] S. Garashchuk and D. J. Tannor, Chem. Phys. Lett. 262, 477 (1996).

[45] W. H. Miller, J. Chem. Phys. 48, 464 (1968).

[46] L. D. Landau and E. M. Lifshitz, Quantum Mechanics (Butterworth-Heinemann, Oxford, U.K., 1999).

[47] J. V. Van Vleck, Proc. Natl. Acad. Sci. U.S.A. 14, 178 (1928).

[48] M. Gutzwiller, Chaos in Classical and Quantum Mechanics (Springer-Verlag, New York, 1990).

[49] S. Garashchuk and V. A. Rassolov, J. Chem. Phys. 121, 8711 (2004).

[50] R. E. Wyatt, C. L. Lopreore, and G. Parlant, J. Chem. Phys. 114, 5113 (2001).

[51] J. C. Burant and J. C. Tully, J. Chem. Phys. 112, 6097 (2000).

[52] O. Prezhdo and V. V. Kisil, Phys. Rev. A 56, 162 (1997).

[53] E. Gindensperger, C. Meier, and J. A. Beswick, J. Chem. Phys. 113, 9369 (2000).

[54] O. V. Prezhdo and C. Brooksby, Phys. Rev. Lett. 86, 3215 (2001).

[55] A. Donoso, D. Kohen, and C. C. Martens, J. Chem. Phys. 112, 7345 (2000).

[56] W. Press, B. Flannery, S. Teukolsky, and W. Vetterling, Numerical Recipes: The Art of Scientific Computing, 2nd ed. (Cambridge University Press, Cambridge, U.K., 1992).

[57] D. J. Tannor and D. E. Weeks, J. Chem. Phys. 98, 3884 (1993).

[58] D. E. Manolopoulos, in Encyclopedia of Computational Chemistry (Wiley, Chichester, 1998), vol. 4, pp. 2600-2708.

[59] C. Leforestier et al., J. Comput. Phys. 94, 59 (1991).

[60] F. Grossmann, Phys. Rev. A 60, 1791 (1999). 